\title{
The Internal Rotation Deficit in Reverse Shoulder Arthroplasty: Can Humeral Rotation Make Difference?
}

\author{
Young-Kyu Kim \\ Department of Orthopaedic Surgery, Gachon University Gil Medical Center, Incheon, Korea
}

Cuff tear arthropathy is the primary indication for reverse shoulder arthroplasty. Early type of anatomic reverse shoulder arthroplasty has fixed and most external placed rotation center. And the long distance of rotation center from glenoid bone causes prosthesis loosening and implants fracture by increased tilting force.

For overcoming these limitations, Grammont and Baulot. ${ }^{1)}$ demonstrated prosthesis design on the basis of his biomechanical work, which has medialized and distalized center of rotation for increasing lever arm of deltoid. This principle of Grammont's prosthesis reduce torque on the glenoid component and increase the deltoid lever arm for overcoming weak or absent rotator cuff musculature. ${ }^{2,3)}$ Early results of RSA have been promising for treatment of cuff tear arthropathy with studies reporting significant improvement in pain relief, active range of motion, and shoulder function. ${ }^{1,47}$ While, there have been documented drawbacks of prosthesis on the basis of Grammont's principle, including scapular notching and loss of shoulder rotation, prosthetic instability. ${ }^{1,4,8,9)}$

To decrease the risk of scapular notching and improve shoulder rotation, several options were available. Lowering the position of the metal baseplate, produced increment of the inferior overhang of glenosphere, were reduced these limitations. ${ }^{1)}$ Despite of this technical overcome, the scapular notching and limited shoulder rotation were still documented.

In the clinical study of the Clinics in Shoulder and Elbow, Park and colleagues suggested that Reverse shoulder arthroplasty provided reliable pain relief and recovery of shoulder function in patients with massive irreparable rotator cuff tear. Especially, they insisted that internal rotation differed significantly from the sacral to the lumbar vertebrae, and external rotation did not change significantly. However, further investigation is recommended to demonstrate this issue, because the study of colleagues was followed-up in the short term.

\section{References}

1. Grammont PM, Baulot E. Delta shoulder prosthesis for rotator cuff rupture. Orthopedics. 1993;16(1):65-8.

2. Boileau P, Watkinson DJ, Hatzidakis AM, Balg F. Grammont reverse prosthesis: design, rationale, and biomechanics. J Shoulder Elbow Surg. 2005;14(1 Suppl S):147S-61S.

3. Terrier A, Reist A, Merlini F, Farron A. Simulated joint and muscle forces in reversed and anatomic shoulder prostheses. J Bone Joint Surg Br. 2008;90(6):751-6.

4. Boileau P, Watkinson D, Hatzidakis AM, Hovorka I. Neer Award 2005: the Grammont reverse shoulder prosthesis: results in cuff tear arthritis, fracture sequelae, and revision arthroplasty. J Shoulder Elbow Surg. 2006;15(5):527-40.

5. Frankle M, Siegal S, Pupello D, Saleem A, Mighell M, Vasey M. The reverse shoulder prosthesis for glenohumeral arthritis associated with severe rotator cuff deficiency. A minimum twoyear follow-up study of sixty patients. J Bone Joint Surg Am. 2005;87(8):1697-705.

6. Seebauer L, Walter W, Keyl W. Reverse total shoulder arthroplasty for the treatment of defect arthropathy. Oper Orthop Traumatol. 2005;17(1):1-24.

7. Sirveaux F, Favard L, Oudet D, Huquet D, Walch G, Molé D. Grammont inverted total shoulder arthroplasty in the treatment of glenohumeral osteoarthritis with massive rupture of the cuff. Results of a multicentre study of 80 shoulders. J Bone Joint Surg Br. 2004;86(3):388-95.

8. Boileau P, Moineau G, Roussanne Y, O'Shea K. Bony increased-offset reversed shoulder arthroplasty: minimizing scapular impingement while maximizing glenoid fixation. Clin Orthop Relat Res. 2011;469(9):2558-67.

9. Nyffeler RW, Werner CM, Simmen BR, Gerber C. Analysis of a retrieved delta III total shoulder prosthesis. J Bone Joint Surg Br. 2004;86(8):1187-91.

Correspondence to: Young-Kyu Kim

Department of Orthopaedic Surgery, Gachon University Gil Medical Center, 21 Namdong-daero 774beon-gil, Namdong-gu, Incheon 405-760, Korea Tel: +82-32-460-3384, Fax: +82-32-468-5437, E-mail: kykhyr@gilhospital.com

Financial support: None. Conflict of interests: None. 